\title{
Testing and simulation of a low-temperature air-source heat pump operating in a thermal buffer zone
}

\author{
Marianne F. Touchie and Kim D. Pressnail
}

Version Post-Print/Accepted Manuscript

Citation Touchie, Marianne F., Pressnail, Kim D. (2014). Testing and simulation

(published version) of a low-temperature air-source heat pump operating in a thermal buffer zone. Energy and Buildings 75, pp. 149-159, doi: 10.1016/j.enbuild.2014.02.015.

Copyright / License

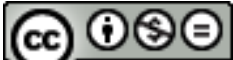

BY Ne ND

This work is licensed under a Creative Commons Attribution-

NonCommercial-NoDerivatives 4.0 International License.

Publisher's Statement The final published version of this article is available via Elsevier at https://dx.doi.org/10.1016/j.enbuild.2014.02.015.

How to cite TSpace items

Always cite the published version, so the author(s) will receive recognition through services that track citation counts, e.g. Scopus. If you need to cite the page number of the TSpace version (original manuscript or accepted manuscript) because you cannot access the published version, then cite the TSpace version in addition to the published version using the permanent URI (handle) found on the record page. 
Testing and Simulation of a Low-Temperature Air-Source Heat Pump Operating in a Thermal Buffer Zone

Marianne F. Touchie (corresponding author), Department of Civil Engineering, University of Toronto

Kim D. Pressnail, Department of Civil Engineering, University of Toronto

Corresponding author contact details: +14166686408, marianne.touchie@mail.utoronto.ca. Department of Civil

Engineering, University of Toronto, 35 St. George Street, Toronto, Ontario, Canada, M5S1A4

\section{Abstract:}

Air-source heat pumps (ASHPs) are commonly used in temperate climates throughout Europe and Asia to provide energy efficient heating and cooling. However, ASHPs have not been widely adopted for heating in colder climates because the coefficient of performance (COP) is lower when outdoor temperatures are colder. While many researchers are working on improving the cold-temperature performance of ASHPs by altering the design of the equipment, this work examines an innovative way to improve performance by operating an existing 'off-the-shelf' ASHP within a thermal buffer zone. This paper shows that operating an ASHP in a thermal buffer zone (TBZ) created by an enclosed balcony space can improve the COP in cold temperatures. An ASHP operating in a TBZ was tested in a climate chamber where the performance was monitored under a variety of climatic conditions. The temperature drawdown of the TBZ and the associated impact on the COP were observed. The TBZ solar heat gain rates required to improve the COP were identified for a range of exterior temperatures. Then, a suite-based energy model was developed and calibrated to simulate the performance of the ASHP operating in the TBZ. The model provided a reasonable prediction of the ASHP performance below $10^{\circ} \mathrm{C}$.

\section{Key words:}

air source heat pump; MURB; energy efficiency; suite-based equipment; energy model 


\section{Introduction}

In terms of occupied land area and provision of services, high-rise multi-unit residential buildings (MURBs) are considered an efficient housing type. However, due to the energy intensity and sheer number of these buildings in large urban areas such as Toronto, Canada, MURB energy-use also has a significant negative impact on greenhouse gas emissions in cities [1]. While Toronto will be used as the context for this study, many urban regions around the world that rely heavily on MURBs for housing stock face similar challenges. One reason why MURBs have high energy intensities is related to space conditioning. Many of these buildings, particularly those constructed in the 1960s and 1970s, are heated with perimeter hydronic or electric radiators and feature a pressurized-corridor ventilation system. Controlling interior conditions in these buildings can be challenging due to the adverse effects of leaky envelopes exacerbated by stack and wind pressure differences that lead to ineffective corridor

pressurization. As a result, in an attempt to regulate their suite temperature in this centrally-controlled system, occupants will often open their windows during cold weather periods [2].

In order to save energy, other strategies for delivering heating, cooling and ventilation air to building occupants are being adopted. One of these strategies is to use suite-based equipment which is preferred by both occupants and property managers alike [3]. Suite-based systems can provide better occupant control of the interior environment. As well, suite-based systems present an opportunity for shifting direct financial responsibility for energy-use from the building owner to the individual occupants. When paired with compartmentalization, or air sealing between suites and corridors, further benefits of suite-based space conditioning can be realized. These include improved fire safety and reduced effects of stack pressure differences and the associated uncontrolled air leakage [4]. In contrast with a typical central HVAC system, where the building is conditioned to a single minimum set point temperature, suite-based systems can also save energy by delivering energy only when and where it is needed.

With the emergence of suite-based equipment in new construction, and the fact that central HVAC systems in many older existing MURBs are reaching the end of their service life, there is an opportunity to replace electric resistance heaters or hydronic baseboard systems and the associated central boilers and pumps with more energyefficient suite-based systems. 
One such system incorporates air-source heat pumps (ASHPs). Located in individual suites, these heat pumps can be as installed as a retrofit strategy to provide demand-based space conditioning. ASHPs are typically used for heating in more moderate climates than Toronto (average annual heating degree days for the period 2010- 2012: 3550 Heating Degree Day (HDD), base $18^{\circ} \mathrm{C}[5]$ ) since the coefficient of performance (COP) declines as the outdoor temperature decreases [6][7]. In order to maintain efficient operation of the ASHP on the coldest days of the year, the evaporator coils must somehow be kept warmer. One opportunity, unique to MURBs, is to make use of the existing balcony space as a Thermal Buffer Zone (TBZ). By enclosing the unconditioned balcony, a volume of air, heated by passive solar gains and heat losses from the apartment, can be used to increase the COP of the ASHP. The work presented here explores how this alternative use of ASHPs operating in cold climates may be able to take advantage of an often underutilized architectural feature: the MURB balcony.

\subsection{Objective}

The aim of this study was to determine the performance benefits of operating an ASHP in a TBZ created by an enclosed balcony space. However, in a relatively small, enclosed space, the compressor unit may significantly draw down the temperature of this zone, so the limitations of this approach were also investigated.

\subsection{Previous work}

In the moderate climates of Europe and Asia, ASHPs are already a popular means of providing energy-efficient heating and cooling [8]. ASHPs have not been widely adopted in colder climates because of significant reductions in the capacity and COP during cold weather. However, the potential for energy savings associated with ASHP use in colder climates has prompted many researchers to investigate how this performance can be improved.

Roth et al. described the strategies used to improve ASHP performance for cold climates [9], including increasing outdoor coil capacities, the use of $\mathrm{CO}_{2}$ for refrigerant, and the use of multiple or modulating compressors, among others. This study also included a comparison of the modeled performance of an ASHP to the performance of a natural gas furnace in various locations with the following annual heating-degree days: $4200^{\circ} \mathrm{C} \cdot d a y s, 3200^{\circ} \mathrm{C} \cdot \mathrm{days}$ and $2600^{\circ} \mathrm{C} \cdot$ days. Depending on furnace efficiency, little or no primary energy consumption savings were found, but in some cases, energy cost savings resulted. This study did not include a discussion of the potential $\mathrm{CO}_{2}$ 
emission reductions associated with switching from natural gas to electricity, which could be viewed as a significant benefit in cities such as Toronto.

Another study [8] showed energy savings of up to $70 \%$ when the performance of an ASHP was compared to electric resistance heating in an apartment. This more direct comparison between electricity-consuming heat sources illustrates the potential energy savings in the study location $\left(3000^{\circ} \mathrm{C} \cdot\right.$ days $)$ and is an encouraging prospect for electrically-heated buildings.

Bush et al. conducted laboratory testing of three ASHPs: a single-speed unit, a variable-speed unit and a variablespeed unit designed for low-temperature conditions [6]. They found that, at temperatures as low as $-15^{\circ} \mathrm{C}$, the rated capacity maintained by the low-temperature unit (70\%) was significantly higher than the rated capacity maintained by the single- and variable-speed units which were $30 \%$ and $55 \%$, respectively. Given the focus on cold climate ASHP performance, a low-temperature variable-speed ASHP was selected for testing in the current study.

In addition to performance testing, there have been many studies suggesting modifications to current ASHP technology to improve cold climate performance. Abdelaziz and Shen described the benefits of multi-stage vapour injection compression cycles for cold climate operation and used an optimization model to suggest changes to capacity and air flow rates for improved performance [10]. Guoyuan et al. developed a prototype of an ASHP with sub-cooling via a supplementary refrigerant circuit which achieved a COP of 2.3 at an ambient temperature of $-15^{\circ} \mathrm{C}[11]$. Later, Guo-Yuan and Hui-Xia showed that an ASHP with a flash-tank could have a higher efficiency than an ASHP with a sub-cooler [12]. Most recently, Jiang et al. showed that the cold temperature performance of an ASHP could be improved by spraying the outdoor unit coils with a glycol solution to prevent frosting [13].

Researchers have also investigated pairing solar collectors with air-to-water heat pumps to supplement performance as cited by Liang et al [14]. Liang et al. also built and tested a solar-assisted heat pump that reduced heat pump energy requirements by $10 \%$ over a heating season with a $40 \mathrm{~m}^{2}$ solar panel.

The motivation behind the work presented here was not to modify an existing ASHP design but rather operate an 'off-the shelf' ASHP in an innovative way by moving the outdoor unit into a TBZ in order to take advantage of solar gains and the heat gain from zones adjacent to the TBZ. 
Passively, gathering solar gains in a TBZ has been investigated by Hix [15] through his Alpha house and Smith [16] through his "Thermal Envelope House." Both of these systems rely on natural convective loops to transport heat. This concept for detached single-family homes was adapted by Pressnail et al. [17] when they introduced the operation of an inter-zonal heat pump to transfer heat from the TBZ to the occupied zone. Dixon et al. [18] furthered this work by developing a more robust model in the building energy simulation software EnergyPlus. They demonstrated that COPs of greater than 3.5 could be achieved by operating the compressor in the TBZ. Stahlbrand and Richman [19] modeled the convective loop generated within a TBZ surrounding a detached singlefamily home using computational fluid dynamics to determine how the location of the ASHP in the convective loop impacted the COP. Thus far, the TBZ/ASHP research at the University of Toronto and Ryerson University has focused on single-family home applications and modeling of the ASHP operation. This study extends the work done to date by conducting laboratory testing of the ASHP in a TBZ and developing a calibrated energy model of the ASHP performance. Additionally, the TBZ/ASHP concept is applied for the first time to a common urban building type in need of retrofit: the high-rise MURB.

\subsection{Approach}

Since Toronto is a heating-dominated climate, the focus of this investigation was on the performance of the ASHP system during winter conditions. In the first part of the study, the baseline "exterior" performance of the ASHP was established by testing it at a range of cold ambient temperatures in a laboratory climate chamber. Then, the ASHP was tested in a TBZ to observe how the TBZ temperature was drawn down by the ASHP operation and the associated changes in COP and power draw were compared to the baseline performance. Next, these TBZ tests were repeated by supplying different rates of heat gain to the TBZ using electric resistance heaters. The different heat supply rates were used to simulate varying levels of sensible heat gain to the TBZ from solar gains and heat gains from the adjacent apartment.

The second part of this study involved the development of an energy model calibrated using the laboratory results in order to simulate the performance of the ASHP operating in the TBZ. 


\section{Laboratory Testing}

This section outlines the laboratory set up including the construction of various zones, the selection of HVAC and control equipment, and an explanation of the test procedure.

\subsection{Laboratory apparatus}

Testing of the ASHP in a TBZ required construction and instrumentation of five different zones in the climate chamber of the Building Science Laboratory at the Department of Civil Engineering, University of Toronto, as shown in Figure 1.

A model apartment unit with an adjacent enclosed balcony was constructed. A Warm Room zone surrounding the Apartment was maintained at the same temperature as the Apartment $\left(23^{\circ} \mathrm{C} \pm 1^{\circ} \mathrm{C}\right)$. In this way, the Warm Room facilitated the measurement of the heat required to maintain the Apartment set point temperature. By maintaining the Warm Room at the same temperature as the Apartment, any heat that was added to the Apartment only flowed between the Apartment and the Balcony.

The bounds of the climate simulator constrained the zone sizes, particularly the Apartment. So, while the Balcony dimensions ( $2.5 \mathrm{~m}$ by $1.4 \mathrm{~m}$ ) were based on those of a typical Toronto MURB, the Apartment was much smaller than an actual apartment. The wall area of the apartment adjacent to the balcony was $5.5 \mathrm{~m}^{2}$ compared with the wall area of an example two-bedroom apartment which was exposed to the exterior $\left(33 \mathrm{~m}^{2}\right)$. This resulted in Apartment heating loads which were significantly less than a typical two-bedroom apartment in Toronto. More realistic Apartment heating loads were generated by providing a balanced air exchange between the Apartment and the Cold Room. In other words, the heating load was artificially increased by introducing a chilled air stream into the Apartment. To do this, a modified energy recovery ventilator (ERV) was installed in the Apartment. The ERV was modified by removing the core and then constructing two ducts. One duct ran from the Cold Room air inlet to the Apartment supply air outlet and one duct ran from the Apartment return air inlet to the Cold Room exhaust air outlet. Air flows in the two ducts were balanced to minimize uncontrolled air leakage between zones. Using the maximum ERV fan speed of $61 \mathrm{~L} / \mathrm{s}$, Cold Room air was supplied to the Apartment. Depending on the Cold Room temperature, this resulted in an estimated increase in the Apartment heating load of between $1 \mathrm{~kW}$ to 
1.6kW. For comparison, the two-bedroom apartment on which the laboratory apparatus was based would lose about $1.3 \mathrm{~kW}$ at an exterior temperature of $1^{\circ} \mathrm{C}$ (based on $18.5 \mathrm{~m}^{2}$ of remaining exposed wall area after enclosing the balcony). Therefore, this approach was thought to reasonably represent the heating load of the larger space in an actual apartment. Additional transmission losses from the Apartment to the Balcony were estimated to be between $0.1 \mathrm{~kW}$ and $0.5 \mathrm{~kW}$ depending on the Balcony temperature during each particular test. Thus, the combination of transmission losses and the balanced supply of cold air led to an Apartment heating load that varied between $1.1 \mathrm{~kW}$ and $2.1 \mathrm{~kW}$. The indoor unit of the ASHP, shown in Figure 2, was located in the Apartment to meet these heating loads. The rated capacity range of the ASHP in heating mode was between $1.3 \mathrm{~kW}$ and $3.52 \mathrm{~kW}[20]$.

A guard box surrounding the outdoor unit of the ASHP, as shown in Figure 3, was constructed to dampen the effects of the climate chamber chiller cycles on the ASHP compressor. During the original experimental design and planning stages, following the baseline performance tests, the outdoor unit was to be moved from the guard box into the Balcony zone adjacent to the Apartment zone. However, after construction and installation of the ASHP, it was decided that, in order to minimize movement of the refrigerant lines, the compressor would remain in the guard box for all of the tests. Therefore the guard box also served as a model TBZ. Note that, as the guard box is separate from the Apartment as shown in Figure 1, the operation of the outdoor unit of the ASHP did not directly affect the temperature of the Balcony or the magnitude of the Balcony heat gains from the Apartment. For the remainder of this paper, the term "TBZ" will refer to the zone created by the guard box surrounding the outdoor unit of the ASHP. The limitations of this approach are discussed in Section 5.

\subsection{Equipment Selection}

This section outlines the specifications of the ASHP tested as well as all other temperature control and metering equipment used to determine the ASHP performance.

\subsubsection{ASHP}

A ductless mini-split ASHP was chosen because, in a retrofit scenario, refrigerant lines are easier to install than new duct work. A variable-speed low-temperature ASHP with a swing compressor was selected. The ASHP was 
manufactured by Daikin Industries Ltd., and has a rated COP of 4.46 in heating mode [20]. According to the manufacturer's specifications, the rated COP was determined using a flow rate of $198 \mathrm{~L} / \mathrm{s}$. However, operating the ASHP with this air flow rate resulted in excessive cycling because the relatively small Apartment heating load was met quickly, even after maximizing the delivery of Cold Room air using the modified ERV. Therefore, the indoor unit fan of the ASHP was operated at the low speed setting $(85 \mathrm{~L} / \mathrm{s})$ so that longer periods of steady state behaviour could be observed. Thus, for all laboratory tests, the ASHP was operated on the low fan speed which, in turn, contributed to a lower COP than specified by the manufacturer. However, the purpose of the laboratory testing was not to verify the manufacturer's COP but rather to explore the effect on COP of the ASHP operation in an enclosed space. The ASHP set point temperature was adjusted between $23.5^{\circ} \mathrm{C}$ and $25^{\circ} \mathrm{C}$ in order to keep the apartment zone temperature as close to $23^{\circ} \mathrm{C}$ as possible for all of the tests.

\subsubsection{Temperature Control Equipment}

Various equipment was required to control the temperatures in the test apparatus. This included thermocouples to measure temperature, a data acquisition unit to collect temperature data from the thermocouples, and a computer to process the temperature readings and execute the temperature control algorithms. To provide radiant heat to the zones, electric resistance heaters were controlled by these algorithms. Climate chamber cooling was provided by a chiller located in the Cold Room.

Air temperatures were measured using Type T thermocouples. The thermocouples were calibrated using a Thermoelectric Pronto 200 thermocouple reader which can measure temperatures between $-200^{\circ} \mathrm{C}$ and $400^{\circ} \mathrm{C}$ and is accurate to $\pm 0.2^{\circ} \mathrm{C}[21]$. The thermocouples were located in pairs approximately $0.3 \mathrm{~m}$ from the ceiling and the floor in various positions throughout each zone. Thermocouples were also located in the air streams of the ASHP and the modified ERV.

A Hewlett Packard data acquisition (DAQ) unit was used to read the thermocouples every 15 seconds and to provide input to a computer program (HTBasic) which controlled relay switches for the fans and heaters in each zone. Average zone temperatures were then calculated and used by the computer program to determine if the heaters should be turned on or off for each 15-second period. Heating was provided by a combination of fans with 
integrated heaters and radiant baseboard heaters. The integrated fan heaters had a high and low setting of $1230 \mathrm{~W}$ and $770 \mathrm{~W}$, respectively. The baseboard heater had a maximum capacity of $1440 \mathrm{~W}$ but was connected to a voltage transformer in order to fine tune the heat supplied to the TBZ for each test. Fans were also used to circulate air to promote more uniform zone temperatures.

Figure 4 shows a schematic of the control loops that were used to maintain the required zone set point temperatures.

\subsubsection{Measuring devices}

In addition to the real-time temperature readings required for zone control, the electricity consumption of the fans, the heaters and the ASHP was metered. To minimize uncontrolled air leakage, spot measurements of differential air pressures were taken to ensure that the air pressure was balanced between zones (less than 0.5Pa difference). The magnitude of the air flows from the ASHP and ERV was also measured. Then, these air flow rates were used with the relevant air stream temperatures to estimate the heat delivered to, and extracted from the Apartment zone. Specifications of the equipment used to measure these various parameters are provided in Table 1.

\subsection{Test Procedure}

The testing began with the establishment of the baseline performance of the ASHP. This was followed by testing to determine how the outdoor unit of the ASHP would perform in a confined space or TBZ. Then, various rates of heat gain were applied to the TBZ to determine the potential COP improvement with the addition of solar gains and apartment heat gains to the TBZ.

The general test procedure was similar to the steady state and cyclic heating mode tests in ANSI/AHRI Standard 210/240-2008: Performance Rating of Air-Source Heat Pump Equipment. As described, for all tests, the outdoor unit of the ASHP was located in the guard box which served as the TBZ, as well.

The base case tests were intended to represent the typical ASHP operation with the outdoor unit operating outside of the building envelope. This condition, where the exterior acts as an infinite heat source or sink, was 
achieved by maintaining a constant TBZ temperature during compressor operation. A fan drawing Cold Room air into the TBZ and a heater located in the TBZ, as shown in Figure 3, operated alternately to maintain this steady TBZ temperature (see Control Cycle 1 in Figure 4).

After establishing the baseline ASHP performance, further tests were used to explore how the ASHP would perform while operating in a limited heat source or sink environment such as a TBZ. Tests were conducted with no heat added to the TBZ to simulate the case with no solar gains. Then, different constant rates of heat gain were applied to the TBZ to simulate varying levels of solar heat gain. Figure 5 shows the rates of heat gain applied to the TBZ in each group of Cold Room temperature tests. For comparison, it also shows the average rate of heat applied to maintain a steady TBZ temperature during the baseline testing.

To determine the ASHP performance in each test, power meter and air temperature readings were collected along with periodic air flow measurements. The availability of these data allowed for the calculation of the instantaneous COP of the ASHP using Equation 1.

Equation 1

$C O P=\frac{\text { Heat delivered by } A S H P}{\text { Power supplied to } A S H P}=\frac{\rho C Q\left(T_{s}-T_{r}\right)}{q_{s}}$

Where

$\rho=$ density of air leaving indoor unit of ASHP $\left(\mathrm{kg} / \mathrm{m}^{3}\right)$

$\mathrm{C}=$ heat capacity of air $(\mathrm{kJ} / \mathrm{kgK})$

$Q=$ flow rate of air leaving indoor unit of ASHP (L/s)

$\mathrm{T}_{\mathrm{s}}=$ temperature air supplied by the indoor unit of the ASHP $\left({ }^{\circ} \mathrm{C}\right)$

$\mathrm{T}_{\mathrm{r}}=$ temperature of return air entering the indoor unit of the $\mathrm{ASHP}\left({ }^{\circ} \mathrm{C}\right)$

$\mathrm{q}_{\mathrm{s}}=$ power supplied to ASHP (W)

All tests were conducted at four different Cold Room temperatures $\left(6^{\circ} \mathrm{C}, 1^{\circ} \mathrm{C},-4^{\circ} \mathrm{C}\right.$ and $\left.-8^{\circ} \mathrm{C}\right)$ and run for a minimum of two hours.

\section{Laboratory results}

Tests were carried out in order to investigate how an ASHP would perform with the outdoor unit located in an enclosed space. It was not known how a rapid change in the temperature of the TBZ would affect the COP. In an 
effort to characterize the ASHP performance in a TBZ, COPs were first calculated using Equation 1 and the methods described in Section 3.1. Then, the influence of the TBZ temperature (Section 3.2), the heat gain rate (Section 3.3) and the Apartment heating load (Section 3.4) on the COP were assessed. Finally, ASHP power trends were explored in Section 3.5 where the influence of TBZ temperature on power draw is presented.

\subsection{Calculation of COP}

Variable-speed ASHPs have a control algorithm that varies compressor and fan speeds with changing conditions. Therefore, truly steady state tests can be only conducted with the aid of a controller provided by the manufacturer. This controller can be used to override the ASHP control algorithm and operate the compressor and fan at a constant speed for the test period. In this study, without access to the manufacturer's controller, attempts were made to determine the Steady State COP during periods when the variable-speed compressor of the ASHP operated at a relatively constant speed. These periods were identified by determining regions when the rate of energy consumption was relatively constant, as denoted by the shaded regions in Figure 6 .

However, during the course of each test, the "steady state" periods were often short and infrequent. These irregular power consumption patterns meant that choosing a steady state period from the test data was not a simple task.

The Steady State COP was determined from the average of the instantaneous COPs, calculated using Equation 1, from each five-second interval in a given steady state period. The average of the instantaneous COPs from the steady state section was then determined. While attempts were made to choose the most representative steady state period, power consumption irregularity meant that even within a 'steady state' section, the instantaneous COPs varied between 0.2 and 0.36 standard deviations from the mean.

The magnitude and frequency of the variations in power consumption were primarily attributed to oscillation between the compressor speeds required to maintain the set point temperature. Other, larger variations were attributed to compressor cycling when the set point temperature was reached or when defrosting of the outdoor unit was required, as confirmed by the zone temperature readings. This cyclic operation is more representative of actual operation, and thus it was the primary interest for this investigation. 
To assess ASHP performance during the entire test period including periods of cycling, an Overall COP was developed. The Overall COP was calculated by dividing the total heat delivered by total power drawn over the entire test period. This is similar to the way a heating seasonal performance factor (HSPF) is calculated. Unlike the HSPF, the Overall COP is presented with the same units $(\mathrm{kW})$ in both the numerator and the denominator. The Overall COPs will be compared to the Steady State COPs in Section 3.2.

\subsection{Influence of TBZ Temperature on COP}

In heating mode, the COP of the ASHP increases when the temperature to which the outdoor unit is exposed increases. This is because there is more energy available for transfer from the outdoor unit to the indoor unit. Figure 7 shows both the Overall and the Steady State COP as determined from each test as well as a plot of the COP based on the ASHP manufacturer's data for the same interior set point temperature and range of Cold Room temperatures.

Figure 7 reveals that the Overall and Steady State COPs are quite similar. It was expected that the Overall COPs would be lower than the Steady State COP given the cyclic ASHP operation throughout each test, but this is not what the authors found. Part of the reason for the similarity of the two measures of COP could be attributed to the period immediately after the compressor had switched off. By observing the temperature of the supply air after the compressor had switched off, it appeared that the refrigerant was still circulating and thus delivering heat for a short period when there was no power draw from the ASHP. With no power draw, and yet with heat still being supplied, the instantaneous COP increased dramatically, approaching infinity during these short periods.

However, there are some differences between the Overall and Steady State COPs. The steady state data, as shown in Figure 7, exhibit greater variation. This may be attributed to the difficulty in choosing steady state periods for some of the tests.

As discussed, the rated COPs from the manufacturer were determined using an indoor air flow rate of 198L/s [20] while the laboratory tests were conducted at a lower fan speed of $85 \mathrm{~L} / \mathrm{s}$, for reasons outlined in Section 2.2.1. Therefore, as expected [26], the reduced airflow during the laboratory testing reduced the heating capacity of the 
ASHP and the resulting COP. However, the rate of change in COP with increased exterior temperatures is almost identical to the manufacturing data as shown by the equations of the lines of best fit in Figure 7.

As the purpose of this investigation was not to determine the absolute COPs but rather the incremental improvements in COP with the changing temperature of the TBZ, the difference in the COP values between the laboratory data and the manufacturer's test data is not of concern. The computer energy model, described in the sections that follow, has been calibrated to the COP trend generated by the laboratory data. However, since the rate of change of the COP with temperature is the same as shown in Figure 7, the rated COP in the model can simply be adjusted as needed for future modeling efforts.

Overall, regardless of which measure of COP one chooses, Figure 7 reveals that the change in TBZ temperature had the anticipated effect on the coefficient of performance of the ASHP.

\subsection{Influence of TBZ Heat Gain Rate on COP}

By operating the ASHP in a TBZ, the temperature of the enclosed space is drawn down as heat is extracted. Gains to the TBZ such as those from the sun can offset this temperature decline. However, if heat is extracted at a rate faster than it is replenished, the temperature of the TBZ will drop below the exterior temperature and thus, there would be no advantage to operating the ASHP in the TBZ. To determine the frequency of this condition, the test results were examined to determine the TBZ heat gain rate and associated TBZ temperature at which the ASHP COP was improved compared to operation at ambient outdoor conditions.

Figure 8 shows the TBZ heat gain rate at which it becomes advantageous to operate the ASHP when the average Cold Room temperature is $1^{\circ} \mathrm{C}$. This heat gain rate, where operating the ASHP in a TBZ becomes advantageous, can be found by determining the point where the TBZ temperature is equal to or greater than the exterior or Cold Room temperature.

The secondary y-axis in Figure 8 shows the proportion by which the COP improves with changes to the TBZ temperature. This COP improvement was determined by Equation 2.

Equation 2 


$$
\% \text { improvement of } C O P=\frac{\left(C O P_{i}-C O P_{\text {base }}\right)}{C O P_{\text {base }}}
$$

Where:

$\mathrm{COP}_{\mathrm{i}}=$ Overall COP from a particular test

$\mathrm{COP}_{\text {base }}=$ hypothetical COP when the TBZ temperature matches the Cold Room temperature

The "base" COP in Equation 2 is a hypothetical COP that occurs at the point where the TBZ temperature is equal to the Cold Room temperature as shown in Figure 8. This base COP was determined for each Cold Room temperature in order to assess the relative improvement or deterioration of COP with TBZ temperature changes. The base COP is determined by finding the rate of heat gain where the TBZ temperature equals the exterior temperature and then locating the corresponding COP using the equation from the Overall COP line shown in Figure 7.

This analysis was repeated for all of the tests conducted. For Cold Room temperatures above $-5^{\circ} \mathrm{C}$, it was more advantageous to operate the ASHP within the TBZ when the rate of heat gain is between $830 \mathrm{~W}$ and $1000 \mathrm{~W}$. However, at $-10^{\circ} \mathrm{C}$, it was more advantageous to operate the ASHP within the TBZ when the rate of heat gain is $740 W$.

To provide perspective, these results are put into the context of the amount of solar heat gain received by the Toronto MURB on which the balcony zone was modeled. Assuming the balcony enclosure had a glazed area similar to the existing exterior wall $\left(2.5 \mathrm{~m}^{2}\right)$, the window between the exterior and the balcony space on the south side of the building would receive at least $740 \mathrm{~W}$ of solar gains for $18 \%$ of the heating season hours and at least $1000 \mathrm{~W}$ for $8.8 \%$ of the heating season hours. In other words, it would only be advantageous to operate the ASHP within the TBZ during these times. However, the assumed $2.5 \mathrm{~m}^{2}$ window size on which this calculation is based is rather modest. In reality, the glazed part of the balcony could be extended from floor to ceiling to maximize solar gains thereby potentially doubling the estimated number of hours for which operation of the ASHP in the TBZ would be beneficial.

As expected, the COP of the ASHP increased with heat added to the TBZ. With the fenestration ratio contemplated here, the proportion of time that operation of the ASHP in the TBZ is advantageous is limited. While the aim of this 
work is not to optimize the balcony enclosure design, there is potential for further improvement in performance with changes to the glazing area.

\subsection{Influence of Apartment heating load on COP}

With the balanced air exchange between the Apartment and the Cold Room, variations in the Cold Room temperature led to changes in the Apartment heating load. To determine the influence, if any, of changes to the Apartment heating load on the COP, tests with similar TBZ temperatures were compared with different Apartment heating loads. By comparing groups of similar TBZ temperatures, the effect of different ambient temperatures on the compressor could be minimized. As expected, for a given TBZ temperature, the magnitude of the Apartment heating load does not have a significant impact on the COP because the variable-speed compressor is able to adjust to a range of heating capacities. However, in an actual application, the ASHP capacity must be appropriately selected for the range of heating loads required for the particular space.

\subsection{Power consumption variance}

The variability in power consumption observed during testing, which perhaps indicates frequent oscillation between compressor speeds, was unexpected and required investigation. With the compressor operating in the TBZ, two factors were thought to influence the ASHP power consumption variance: the average TBZ temperature and TBZ temperature variance. A multi-variable regression analysis was conducted to determine how each of these parameters affected the power consumption variance. The equation for the curve that relates these parameters is shown in Equation 3 with an adjusted coefficient of determination $\left(R^{2}\right)$ value of 0.47 .

Equation 3

$$
\sigma_{A S H P \text { Power consumption }}^{2}=22289+6765 \sigma_{T_{T B Z}}^{2}-3651 T_{T B Z}
$$

Where

$\sigma^{2}=$ Variance

TTBZ $=$ TBZ Temperature $\left({ }^{\circ} \mathrm{C}\right)$ 
As expected, the variance of the ASHP power consumption is positively affected by variance in TBZ temperature, since the compressor speed must adapt to changes in the TBZ temperature. However, it is important to note that the TBZ temperature changes are actually caused by the compressor operation, so these variables are interdependent. Additionally, there is a negative correlation between the TBZ temperature and the power consumption variance, indicating that the power consumption is more variable as the TBZ temperature drops. Furthermore, by observing the magnitude of the impact of each term on the power consumption variance, it is evident that the fluctuation of the TBZ temperature appears to affect the ASHP power consumption variance more than the absolute temperature of the TBZ.

This relationship between temperature, temperature variance and power consumption variance will be different for other ASHP models because the control algorithm, which is unique to each model, will dictate how the compressor responds to changes in the operating conditions.

To further explore this relationship, Figure 9 shows the influence of TBZ temperature on the variance of the ASHP power consumption. At the outset of the testing, it was suspected that the ASHP power consumption would be more erratic during tests where the TBZ temperature was drawn down by the compressor operation. It was thought that these rapid temperature drops would cause the compressor to change speeds frequently. In reality, the tests, where no heat was added to the TBZ and where the greatest temperature draw down was observed, exhibited the highest variances in each Cold Room temperature group, but not the highest variance overall. This indicates that absolute TBZ temperature is a contributing factor in addition to TBZ temperature variance. This was confirmed by the multivariable regression analysis presented above. Conversely, it was believed that the tests where the TBZ temperature was kept reasonably constant would result in the most steady state compressor operation as indicated by power consumption variance. However, the tests with a steady TBZ temperature did not exhibit a lower power consumption variance than the other tests in each Cold Room temperature group. This reveals that operating the compressor of the ASHP in a confined space does not appear to significantly increase compressor speed oscillation or cycling. 


\section{Energy modelling}

Analysis of the laboratory test results in Section 3 provided insight into how to best calibrate an energy model. In this section, an energy model was developed and calibrated so that the laboratory results could be incorporated into a whole-building simulation in the future. By developing such a model, an ASHP operating in a TBZ could be modeled to determine the impact of this proposed retrofit option on the energy consumption of an entire building. The building energy simulation program EnergyPlus was selected to model the laboratory testing conditions, primarily because code modifications to allow a heat pump to function between two zones had already been developed [18].

This section begins with a brief discussion of the code modifications followed by details of the model set up. Then, the model calibration technique is outlined. Finally, a comparison of the modelling results to the laboratory data is presented.

\subsection{Code modifications}

For the work done by Dixon et al., described in Section 1.2, source code modifications to EnergyPlus were required. In 2009, Dixon et al. [18] modified the version 4.0.0 source code of EnergyPlus to enable the heat pump object to operate between two zones as opposed to pumping heat to and from the exterior. However, these code changes were not incorporated into future versions of EnergyPlus. At the time of writing, the current version of EnergyPlus was 8.0.0. Thus, the first step in modeling the laboratory performance of the ASHP was to transfer the previous source code modifications from version 4.0.0 to the current 8.0.0 version. Once the ASHP operation could be modeled between two zones, a model of the laboratory set up was developed.

\subsection{Model set up}

The energy model was constructed as a simplified version of the laboratory set up with three adjacent zones, as shown in Figure 10. The thermal resistance values shown here were determined following calibration of the model described in Section 4.3. The ceiling and floor of each zone was assumed to be adiabatic. 
In the model, two thermostats were used: one in the Apartment to control the ASHP and one in the TBZ to control the electric resistance baseboard heater. The capacity of the electric resistance heater in the TBZ was then modified to match the metered energy consumption from the actual TBZ heaters in the laboratory during each particular test.

Following establishment of the model geometry, construction and basic controls, a challenge with the ASHP component of the model was encountered. The source code modifications enabled a working single-speed ASHP model using the 'AirLoopHVAC:UnitaryHeatPump:AirToAir' object in EnergyPlus. However, the laboratory tests were conducted with a variable-speed ASHP. Time and resource constraints did not allow for further development of the code required for a working variable-speed model so other methods of using the existing code modifications were sought. Various means of addressing this issue were explored in order to model the laboratory performance.

In the end, the strategy pursued was to compare the single- and variable-speed performance curves to determine if the model was still usable for calibration using the laboratory data. There are four performance curves in the energy modeling software (Equation 4, Equation 5, Equation 6, Equation 7) that are influenced by the manufacturer test data [27].

Equation 4

$$
\text { TotCapTempModFac }=a+b\left(T_{d b, i}\right)+c\left(T_{d b, i}\right)^{2}+d\left(T_{d b, o}\right)+e\left(T_{d b, o}\right)^{2}+f\left(T_{d b, i}\right)\left(T_{d b, o}\right)
$$

Equation 5

$$
\text { TotCapFlowModFac }=a+b(f f)+c(f f)^{2}
$$

Equation 6

$$
\text { EIRTempModFac }=a+b\left(T_{d b, i}\right)+c\left(T_{d b, i}\right)^{2}+d\left(T_{d b, o}\right)+e\left(T_{d b, o}\right)^{2}+f\left(T_{d b, i}\right)\left(T_{d b, o}\right)
$$

Equation 7

$$
\text { EIRFlowModFac }=a+b(f f)+c(f f)^{2}
$$

Where

TotCapTempModFac $=$ Total heating capacity modifier curve as a function of temperature 
TotCapFlowModFac $=$ Total heating capacity modifier curve as a function of flow fraction

EIRTempModFac $=$ Energy Input Ratio (EIR) modifier curve as a function of temperature

EIRFlowModFac $=$ EIR modifier curve as a function of flow fraction

$\mathrm{Tdb}, \mathrm{i}=$ Apartment dry bulb temperature $\left({ }^{\circ} \mathrm{C}\right)$

$\mathrm{Tdb}, \mathrm{o}=\mathrm{TBZ}$ dry bulb temperature $\left({ }^{\circ} \mathrm{C}\right)$

$\mathrm{ff}=$ flow fraction $=$ actual indoor unit air flow rate $/$ rated indoor unit air flow rate

Given particular air flow rates and temperature conditions, the equation for each curve produces a factor. The "TotCap" factors are applied to the rated capacity while the "EIR" factors are applied to the Energy Input Ratio (EIR)(the inverse of the rated COP), to produce an estimate of the actual capacity or EIR at the particular temperature and flow conditions.

There was little difference between the single- and variable-speed curves for the relationship between the temperature of the air entering the outdoor unit and the capacity (Equation 4) with a coefficient of variation of the root mean squared error (CVRMSE) of $1.5 \%$. Thus, a single-speed heat pump could be used to model this aspect of the variable-speed ASHP performance.

To plot the relationships between the mass flow rate of the entering air and the capacity (Equation 5) and EIR (Equation 7), only two flow rates were available from the variable-speed ASHP manufacturer. Due to this lack of data, these relationships appeared linear. When comparing the single and variable-speed curves, the flow-EIR relationship was similar (CVRMSE of $8.3 \%$ ) while the flow-capacity relationship was not (CVRMSE of 33\%). However, all laboratory tests were operated at a constant interior air flow rate. Therefore, once the model was calibrated, the difference between these curves is negligible because the air flow rate does not change. The only issue here is that the model input air flow determined by calibration may not be the same as the flow rate measured in the laboratory. 
The most significant concern was the substantial difference between the single- and variable-speed air temperature-EIR relationship over the range in temperatures shown in Figure 11. Essentially, the COP of the variable-speed ASHP was less affected by exterior temperature than the single-speed ASHP. Recognizing that the performance of the single- and variable-speed ASHPs near the intersection of the two lines would be similar, the laboratory test series with a TBZ temperature range that centred around this intersection (the $1^{\circ} \mathrm{C}$ test series) was chosen for the model calibration. Ideally, the model would have been calibrated with all of the laboratory data, but without a variable-speed model, full calibration of the model was not possible at the upper and lower extremes of the range of temperatures tested. While less than ideal, the calibration was still carried out for illustrative purposes. An alternative strategy will have to be developed for future modeling efforts including development of a whole-building model.

\subsection{Model Calibration}

To begin calibrating the model, all known data were entered into the model including the Apartment and Cold Room temperatures as well as the TBZ heat gain rates. The Apartment temperature from the laboratory testing data was incorporated into the thermostat schedule. Also, a custom weather file was generated from the laboratory temperature data so that the conditions in the model were the same as the conditions in Cold Room. The rate of heat applied by the TBZ heaters, shown in Figure 5, was determined from the electricity metering during the laboratory testing, as described in Section 4.2.

The model variables including infiltration, wall conductivity and ASHP coil capacity as well as rated COP were adjusted until the modeled data approached the measured test data including ASHP power consumption, air temperatures, and heat gain and loss rates. The calibration process involved a trade-off between matching the model to the measured COP or the TBZ temperature. Priority was placed on calibration to the COP because this parameter would have the greatest affect on building energy-use in future modeling efforts.

During the calibration process key parameters were extracted from the model results and compared with the available laboratory data. Following calibration, the ASHP parameters that yielded the best fit to the laboratory data were a rated heating capacity of $2200 \mathrm{~W}$ and a rated COP of 2.7. 


\subsection{Model results compared with laboratory results}

Once the model was calibrated with one test, all remaining test data from the $1^{\circ} \mathrm{C}$ series were compared to the model to determine if the changes in zone temperature and COP could be accurately predicted. As shown in Figure 12 , the model was able to reasonably predict the Balcony temperature. As discussed in Section 2.1 , the Balcony was not used for its originally intended purpose: a TBZ in which the ASHP could operate. Instead, the temperature of the Balcony zone simply floated between the Apartment and Cold Room temperatures. The temperature of the actual TBZ, or guard box, however, was not accurately predicted by the model. The TBZ temperatures were overestimated with increasing rates of heat added to the zone.

This could be due to the small volume of air and the associated complex heat transfer relationships in the TBZ space. Generally, the model results from the colder conditions appear to match the laboratory COP data well. However, in warmer temperature tests, the model underestimates the COP, as shown in Figure 13. To address this deviation in the warmer temperature region, review and refinement of the source code is required. Nevertheless, the model data reasonably matched the laboratory results in the colder temperature region, which is the focus of this study.

\section{Discussion and Recommendations}

The work presented here was comprised of two main parts: laboratory testing of an ASHP operating in a TBZ and then the calibration of an energy model of the laboratory apparatus using the data collected. This section first discusses some of the sources of error in the laboratory work and opportunities to refine the results with future testing. This is followed by a discussion of how the results of the laboratory work can be applied. Finally, the energy modeling challenges are summarized.

Despite attempts to extract "steady state" periods from the test data, the ASHP power consumption was not constant during these periods. In a given "steady state" period, the compressor still appeared to oscillate between speeds as indicated by the ranges of power consumption within each steady state period (from $25 \mathrm{~W}$ up to $400 \mathrm{~W}$ ). Obtaining a controller from the manufacturer would likely result in steady state tests with more constant compressor speeds. This would have allowed for calculation of a true steady state COP. Without such a controller 
and the ability to determine true steady state behaviour, the Overall COP is the best measure of COP performance. Had additional data such as refrigerant flow rates been collected, alternative parameters such as the ratio between exergy destruction and total exergy input to the ASHP could have also been determined [28].

If a larger artificial heating load had been applied to the Apartment zone, the indoor fan speed and set point temperature could have been set higher in order to observe more realistic COPs. This would have also increased the capacity of the ASHP. In the current work, most of the testing occurred at the lower end of the capacity range for this particular ASHP resulting in a lower estimate of the COPs compared to those that could be expected in an actual apartment application.

The overall objective of the laboratory testing was to show whether or not the COP of the ASHP could be improved by locating the outdoor unit in a TBZ. In Section 3.3, it was shown that the impact of a TBZ on the COP of an ASHP is positive if the TBZ is warmer than the exterior temperature and negative if the TBZ is colder than the exterior temperature. In order to maximize the benefit and eliminate the penalty of operation within a TBZ, the ASHP could be designed to extract heat from either the TBZ or the exterior as needed. One possibility would be to supply air to the outdoor unit using a split duct, with one side drawing air from the TBZ and the other drawing air from the exterior. A control algorithm could then be used to operate a motorized damper that changes the air source depending on the temperature differential between the TBZ and the exterior.

For all tests, the outdoor unit was located in the guard box TBZ which was not connected to the Apartment. Had the outdoor unit of the ASHP been moved into the Balcony for the TBZ tests, the influence of the temperature drawdown by the compressor on the Balcony heat gains from the Apartment could have been observed. Since the TBZ was separate from the Balcony, this aspect of the model could not be calibrated. Instead, the Balcony temperature was allowed to float. However, the energy model was capable of simulating the heat flows associated with this arrangement, so this will be explored in future modeling efforts.

The primary goal for developing and calibrating an energy model was to extend the suite-based laboratory testing to a whole-building model in the future. Had the calibrated model functioned as intended, the energy savings associated with replacing traditional hydronic and electric baseboards with suite-based ASHPs operating in TBZs 
could easily be estimated for different buildings in different locations. An alternative method of estimating these energy savings is proposed in Section 6.

\section{Future Work}

Aside from developing the source code necessary to model the variable-speed ASHP operating in a TBZ, the authors propose the development of a tool that can apply factors to the energy consumption outputs of an energy model to approximate the effect of the inter-zonal ASHP.

In this proposed method, the Apartment heating load and solar gains to the Balcony will be simulated using the energy modeling software. The COP of the ASHP will be derived from the trends observed in the laboratory and the Balcony temperature produced by the energy model. Then, the heat losses between the Apartment and the Balcony, and the Balcony and the exterior, can be calculated based on these zone and exterior temperatures. Assuming no heat transfer between adjacent apartments, the heat lost from the Apartment to the Balcony must equal to the sum of the rate of internals gains in the Apartment plus the heat delivered by the ASHP. Using the COP based on the Balcony temperature, the energy input to the ASHP can be determined as a proportion of the apartment heating energy consumption. Finally, the new Balcony temperature can be calculated based on the

energy balance of that zone. By conducting this analysis over a 24-hour period, electricity consumption reduction factors based on different solar heat gains and exterior temperatures can be established. Once applied to the energy consumption of the heaters throughout the building, an estimate of the whole-building energy consumption when operating ASHPs within TBZs can be determined.

\section{Conclusions}

Lower temperature ASHPs show promise for reducing the energy consumption required for heating and cooling in a cold climate such as Toronto. Many researchers have proposed alternative ASHP designs to improve cold temperature performance [9][10][11][14]. However, this study showed that the performance of an off-the-shelf ASHP could be improved by operating it in an innovative way by placing the outdoor unit in a TBZ. The specific 
application contemplated here involves enclosing MURB balconies to create this TBZ while providing suite-based, demand-controlled space conditioning.

Laboratory testing of an ASHP operating in a TBZ was conducted across a range of exterior temperatures and TBZ heat gain rates to characterize performance trends. The rate of change of the COP with the temperature to which the outdoor unit was exposed was found to be the same in the laboratory as the manufacturer data suggested. However, the COPs presented here are lower than the manufacturer data due to several factors including lower interior unit air flow rates. Also established were the required TBZ heat gain rates needed to improve the COP compared to exterior operation. These data can be used in future modeling work. Finally, the variance in the power consumption of the ASHP appears to be correlated with the temperature and the temperature variance to which the outdoor unit was exposed.

Attempts were made to model the laboratory test results so that these suite-level findings could be applied on a whole-building scale in the future. While a working EnergyPlus model was generated, the single-speed ASHP model could not be adequately calibrated to match all of the laboratory data derived from the operation of the variable-speed ASHP testing. Nevertheless, the model reasonably predicted the COPs and the balcony temperatures below $10^{\circ} \mathrm{C}$. However, the model was not a good predictor of the TBZ temperatures.

In order to further the work presented here, the authors have proposed a hybrid approach to modelling this potential retrofit strategy on a whole-building level. The approach includes the development of a spreadsheetbased time series model that uses the solar gains from a whole-building energy model and the COPs from the laboratory data to modify suite-based heating loads by an energy consumption reduction factor. This approach can be used to determine the impact on energy-use of retrofitting an existing MURB with suite-based ASHPs operating in enclosed balconies. 


\section{Acknowledgements}

The authors gratefully acknowledge the equipment provided by Daikin Industries, Ltd. and the assistance provided by Brian Gibbon from Nu-Air Ventilation Systems as well as the funding provided by the National Sciences and Engineering Research Council Engage Grant and the Neil B. Hutcheon Bequest.

\section{References}

[1] M.F. Touchie, C. Binkley, K.D. Pressnail, Correlating energy consumption with multi-unit residential building characteristics in the city of Toronto, Energy and Buildings 66 (2013) 648-656.

[2] Y. Jian, Y. Guo, J. Liu, Z. Bai, Q. Li, Case study of window opening behaviour using field measurement results, Building Simulation, 4 (2011) 107-116.

[3] Finn Projects (Synchronicity Projects Inc.),Survey of In-Suite Space and Domestic Hot Water Heating Systems in Multi-unit Residential Buildings, CMHC Research Report, 2003.

[4] Canada Mortgage and Housing Corporation, Evaluation of Air Leakage Control Measures to Compartmentalize Newly Constructed Suites in a High-Rise Residential Building, CMHC Research Highlight, Technical Series 06-102, 2006.

[5] BizEE, Custom Degree Day Data, 2013. [Internet]. [Cited Dec 2 2013] Available from:

http://www.degreedays.net/\#

[6] J. Bush, R. Domitrovic, A. Amarnath, Low Outdoor Temperature Heat Pump Applications to reduce electric resistance second stage heat, ASHRAE Transactions, 118 (2012) 620-627.

[7] Natural Resources Canada, Heating and Cooling with a Heat Pump, 2004.[Internet]. [Cited June 26 2012] Available from: http://oee.nrcan.gc.ca/sites/oee.nrcan.gc.ca/files/pdf/publications/infosource/pub/home/heatingheat-pump/booklet.pdf

[8] J.E. Bugbee, J. R. Swift, Cold Climate Ductless Heat Pump Performance, Energy Engineering, 110.8 (2013) $47-57$. 
[9] K. Roth, J. Dieckmann, J. Brodrick, Heat Pumps for Cold Climates, ASHRAE Journal, February 2009.

[10] O. Abdelaziz, B. Shen, Cold climates heat pump design optimization, ASHRAE Transactions, 118.1 (2012) 34.

[11] M. Guoyuan, C. Qinhu, J. Yi, Experimental investigation of air-source heat pump for cold regions, International Journal of Refrigeration, 26(1) 2003 12-18.

[12] M. Guo-Yuan, Z. Hui-Xia, Experimental study of a heat pump system with flash-tank coupled with scroll compressor, Energy and Buildings, 40(2008) 697-701.

[13] Y. Jiang, H. Fu, Y. Yao, L. Yan, Q. Gao, Experimental study on concentration change of spray solution used for a novel non-frosting air source heat pump system. Energy and Buildings, 68(2014) 707-712.

[14] C. Liang, X. Zhang, X. Li, X. Zhu, Study on the performance of a solar assisted air source heat pump system for building heating, Energy and Buildings, 43 (2011) 2188-2196.

[15] J. Hix, Buildings in Nature, second ed., John Hix Architect Ltd., [Internet]. [Cited June 2 2013] Available at: http://johnhixarchitect.com/JHA-PUBS/27529-HIX-Book_SecondEdition_medres.pdf

[16] Congress of the United States, Office of Technology Assessment, An Assessment of Technology for Local Development, [Internet], [Cited May 15 2013] Available at: http://ota-cdn.fas.org/reports/8105.pdf pp.55-58.

[17] Pressnail, K.D., Richman, R., Kirsh, A.M., An innovative approach to low-energy building performance using nested thermal envelopes. In: National Building Envelope Council, $12^{\text {th }}$ Canadian conference on building science and technology. Montreal, Canada. 6-8 May 2009.

[18] Dixon, E. Richman, R. Pressnail, K.D., Touchie, M., 2010a. NTED ${ }^{\mathrm{TM}}$ : An innovative design using nested thermal envelopes to achieve significant reductions in energy use. In: Thermal Performance of the Exterior Envelopes of Whole Buildings XI International Conference. Clearwater Beach, United States. 5-9 Dec 2010.

[19] I. Stahlbrand, R. Richman, Simulation of a convective loop for the Nested Thermal Envelope Design ${ }^{\mathrm{TM}}$ lowenergy house, Journal of Building Physics, 36(1), (2012) 57-82. 
[20] Daikin AC Americas, Inc. Engineering Data Split Heat Pump FTXS-L/FDXS-L Series

[21] Thermoelectric. n.d. Pronto 200 Manual.

[22] P3 International. n.d. P4460 Kill A Watt ${ }^{\mathrm{TM}}$ EZ Operating Manual Rev. 0307

[23] Extech Instruments. 2012. Product Datasheet for Dual Input True RMS AC Voltage/Current Datalogger, [Internet][Cited September 25 2013] Available at:

http://www.extech.com/instruments/resources/datasheets/DL160data.pdf

[24] Air Instrument Resources Ltd. n.d. Operating and Calibration Instructions for MP Series Precision Micromanometers.

[25] The Energy Conservatory. 2013. Minneapolis Duct Blaster Specifications. [Internet][Cited September 25 2013] Available at: http://www.energyconservatory.com/products/duct-blaster\%C2\%AE-systems-and-accessories

[26] L. Palmiter, J. Kim, B. Larson, P.W. Francisco, E.A. Groll, J.E. Braun. Measured effect of airflow and refrigerant charge on the seasonal performance of an air-source heat pump using R-410A, Energy and Buildings, 43 (2011) 1802-1810.

[27] U. S. Department of Energy, EnergyPlus Engineering Reference, [Internet][Cited April 28 2013] Available: http://apps1.eere.energy.gov/buildings/energyplus/pdfs/engineeringreference.pdf

[28] C. Coskun, Z. Oktay, I. Dincer, Investigation of some renewable energy and exergy parameters for two Geothermal District Heating Systems, Int. J. Exergy, 8(1) 2011, 1-15.

\section{List of Captions}

Figure 1: Plan view schematic of zones within the climate simulator

Figure 2: Indoor unit of ASHP located in the Apartment

Figure 3: Outdoor unit of ASHP located in a guard box (Thermal Buffer Zone)

Figure 4: Equipment control cycles 
Figure 5: Tests conducted

Figure 6: ASHP power consumption from sample $1^{\circ} \mathrm{C}$ test

Figure 7: Influence of TBZ temperature on COP

Figure 8: Influence of various TBZ heat gain rates on zone temperatures

Figure 9: Influence of TBZ temperature on power consumption variance

Figure 10: Zoning for energy model

Figure 11: Variation in EIRTempModFac with exterior temperature

Figure 12: Comparison between modeled and measured zone temperatures

Figure 13: Comparison between modeled and measured COP 